\title{
Identidad, Ética y Diseño: \\ Un análisis de los múltiples cruces posibles Proyecto de Investigación
}

$\mathrm{N}^{\circ} 8.3$

Equipo de Investigación ${ }^{(1)}$

Marina Matarrese

por Facultad de Diseño y Comunicación, Universidad de Palermo (ARG)

Luz del Carmen Vilchis Esquivel por Universidad Nacional Autónoma de México (MEX)

\begin{abstract}
Resumen: El Proyecto de Investigación 8.3 presenta reflexiones acerca de la articulación entre diseños altamente referenciados en las identidades de distintos grupos y/o minorías, el campo del diseño, la comercialización de objetos de/con diseño y la ética. De allí que se abra un prolífico campo para reflexionar académicamente acerca de los múltiples factores de intersectan esta temática altamente referenciada en los procesos de globalización y glocalización. Otra arista importante es cómo incide la desigualdad: económica, de poder, de género, en el reconocimiento o no de la autoría y a la hora de realizar los reclamos y disputas para tales reconocimientos.
\end{abstract}

Palabras clave: Diseño - Identidad - Desafíos - Desigualdad - Poder - América Latina

[Resúmenes en inglés y portugués en la página 215]

${ }^{(1)}$ Los CVs del Equipo de Investigación pueden consultarse en el Capítulo Directores de Líneas y Coordinadores de Proyectos de esta misma Edición.

\section{Acerca del Proyecto 8.3}

\section{Identidad, Ética y Diseño}

Un análisis de los múltiples cruces posibles

El Proyecto 8.3 Identidad, Ética y Diseño: Un análisis de los múltiples cruces posibles, reúne diversas reflexiones acerca de la articulación entre diseños altamente referenciados en las identidades de distintos grupos y/o minorías, el campo del diseño, la comercialización de objetos de/con diseño y la ética. De allí que se abra un prolífico campo para reflexionar académicamente acerca de los múltiples factores de intersectan esta temática 
altamente referenciada en los procesos de globalización y glocalización. Otra arista importante es cómo incide la desigualdad: económica, de poder, de género, en el reconocimiento o no de la autoría y a la hora de realizar los reclamos y disputas para tales reconocimientos. Se acordó entre la Universidad de Palermo y la Universidad Nacional de México, avanzar en una investigación en la que participan académicos de ambas Instituciones, con la coordinación compartida de Marina Matarrese (UP) y Luz del Carmen Vilchis Esquivel (UNAM), con el fin de impactar en las estrategias de investigación en diseño en Latinoamérica, a través de comparar las diversas experiencias realizadas en Instituciones de Educación Superior de la región con la llevada adelante por la Facultad de Diseño y Comunicación de la Universidad de Palermo. Las reflexiones y los resultados obtenidos en el Proyecto 8.3 Identidad, Ética y Diseño: Un análisis de los múltiples cruces posibles, son continuación de los Proyectos 8.2 Nuevos modos de gestión institucional en la investigación en Diseño y 8.1 Experiencias comparadas: Investigar Diseño en América Latina, en el marco de la misma Línea de Investigación y bajo la misma Directora Marina Matarrese.

Sus principales objetivos son:

- Revisar las convergencias y divergencias de las acciones de los diseñadores en el marco del diseño, la identidad y la ética, haciendo foco sobre los parámetros de la conducta profesional.

- Reflexionar acerca de las miradas éticas acerca de la práctica del diseño como ejercicio profesional en su articulación con pueblos indígenas y con respecto a sus transformaciones históricas.

- Contribuir al desarrollo de las reflexiones teóricas sobre la ética en el diseño, de su ethos y en relación con los desafíos que proponen los tiempos actuales y el mercado a dicho ethos.

- Favorecer los debates en relación al diseño y los desafíos que conlleva éticamente la formación de nuevas generaciones de diseñadores en el ámbito universitario de grado y posgrado.

\section{Acerca de la Línea 8}

Investigar en Diseño dirigida por Marina Matarrese se desarrolla de manera ininterrumpida desde 2015 en la Facultad de Diseño y Comunicación (UP, Argentina), e incluye hasta el momento tres proyectos finalizados el 8.1 Experiencias comparadas: Investigar Diseño en América Latina, el 8.2 Nuevos modos de gestión institucional en la investigación en Diseño, y el 8.3 Identidad, Ética y Diseño: Un análisis de los múltiples cruces posibles, coordinados por Marina Matarrese (UP) y Luz del Carmen Vilchis Esquivel (UNAM). 


\section{Mapa de Áreas y Proyectos}

El Proyecto 8.3 se vincula con todas las carreras de grado y posgrado correspondientes a la Facultad de Diseño y Comunicación.

\section{Productos y Resultados}

\section{a)- Publicaciones}

Cuaderno del Centro de Estudios de Diseño y Comunicación No120. (2020/2021) Investigar en diseño: Identidad, Ética y Diseño. Un análisis de los múltiples cruces posibles. Coordinación Marina Matarrese y Luz del Carmen Vilchis. Año XXIV, Buenos Aires, Argentina. ISSN: 1668-0227. Esta publicación documenta y comunica los resultados alcanzados en el proyecto de investigación 8.3 Identidad, Ética y Diseño. Un Análisis de los múltiples cruces posibles, y a continuación se detallan los autores y artículos contenidos en ella:

Matarrese Marina Laura, Vilchis Luz del Carmen (2020/2021) Introducción. Espacios de la ética y la identidad en el quehacer del diseño (Pp. 11-20)

Medina Robalino, Aylen (2020/2021) Indumentaria indígena: ética, política y diseño. Una mirada sobre el artefacto vestimentario de la mujer chibuleo (Pp. 21-41) Martínez Loera, Ruth Verónica (2020/2021) Creación de la memoria iconográfica de una cooperativa de bordadoras indígenas, un ejercicio ético para el diseñador (Pp. 43-57)

Alvarez Saavedra, Eugenia (2020/2021) Uso del color y patrones geométricos en los diseños Mapuche contemporáneos: Adaptación semiótica en tres casos de estudio comparados (Pp. 59-70)

Alvarez Tamayo, Dora Ivonne (2020/2021) Si la marca fuera una persona: análisis semiótico analógico (Pp. 71-82)

Gómez Romero, Gerardo (2020/2021) Diseño, tipografía y comunicación visual. Ahora todo vale (Pp. 83-100)

Vilchis, Luz del Carmen (2020/2021) Abstracciones filosóficas acerca del ethos del Diseño (Pp. 101-117)

Lezama Galindo, Omar (2020/2021) Reflexiones acerca de la identidad, la ética y la memoria en el diseño gráfico (Pp. 119-137)

Morales González Elia del Carmen, del Moral Zamudio Yésica Alejandra (2020/2021) Consideraciones reflexivas para la acción del diseñador a partir de la ética y la identidad (Pp. 139-156)

Gómez Ordoñez Flor de María, López Colín María Teresa Alejandra (2020/2021) La identidad del Diseñador Gráfico del siglo XXI y la exigencia de la responsabilidad social universitaria (Pp. 157-173)

Ceja Bravo, Leobardo Armando (2020/2021) La otredad subyacente como creencia. Una reflexión ética, política y formativa en diseñadores de posgrado (Pp. 175-185)

Gutiérrez Miranda, Martha (2020/2021) Valores y responsabilidades del Diseño en la era de lo "hiper" (Pp. 187-200) 


\section{b)- Congresos / Coloquios / Plenarios}

V Coloquio de Investigación y Desarrollo en Diseño Latino. Universidad de Palermo, 27 de julio de 2020. XI Congreso Latinoamericano de Enseñanza del Diseño. Semana Internacional del Diseño en Palermo.

En la comisión Investigar en Diseño, se presentaron las reflexiones y conclusiones del proyecto 8.3 Identidad, Ética y Diseño. Un análisis de los múltiples cruces posibles (Finalizado) expusieron: Adriana Vigueras Villaseñor, Flor de María Gómez Ordoñez, María Teresa Alejandra López Colín, Leobardo Armando Ceja Bravo, Dora Ivonne Alvarez Tamayo, Yésica Alejandra del Moral Zamulio, Elia del C. Morales González, Gerardo Gómez Romero, Luz del Carmen Vilchis, María Laura Garrido, Martha Gutiérrez Miranda, Omar Lezama Galindo, Ruth Verónica Martínez Loera, Eugenia Álvarez Saavedra, Aylén Medina, Roberto González, Lucero Ibarra, Luis Alejandro Pérez y Mercedes Martínez.

A continuación se detallan las ponencias presentadas en esta Comisión coordinada por la Directora del proyecto Marina Matarrese:

Adriana Vigueras Villaseñor (México)

Diseño y ética en la identidad del escudo mexicano nacional

Flor de María Gómez Ordoñez y María Teresa Alejandra López Colín (México) La identidad del Diseñador Gráfico del siglo XXI: Entre el ethos y la exigencia de la Responsabilidad Social Universitaria

Leobardo Armando Ceja Bravo (México)

La otredad subyacente como creencia. Una reflexión ética, politica y formativa en diseñadores de posgrado

Dora Ivonne Alvarez Tamayo (México)

Si la marca fuera persona: análisis semiótico analógico

Yésica Alejandra del Moral Zamulio y Elia del C. Morales González (México)

El diseño como generador de identidad desde el pensar en el otro

Gerardo Gómez Romero (México)

Diseño, tipografía y comunicación visual. Ahora ¿̇todo vale?

Luz del Carmen Vilchis (México)

Abstracciones filosóficas acerca del ethos del Diseño

María Laura Garrido (Argentina)

El Diseño Editorial como herramienta social: un proyecto cultural trilingüe

Martha Gutiérrez Miranda (Argentina)

Valores y responsabilidades del Diseño en la era de lo "hiper"

Omar Lezama Galindo (México)

Identidad, ética y diseño. Un análisis de los múltiples cruces posibles

Ruth Verónica Martínez Loera (México)

Creación de la memoria iconográfica de una cooperativa de bordadoras indígenas un ejercicio ético para el diseñador

Eugenia Álvarez Saavedra (Chile)

Emprendedoras de cultura Mapuche en el diseño con motivo gráfico. Relatos de las nuevas generaciones en la región de La Araucanía en Chile 
Aylén Medina (Ecuador)

Indumentaria indígena: entre la política y el diseño. Una mirada del artefacto vestimentario de la mujer chibuleo en el Ecuador (década de 1990)

Roberto González, Lucero Ibarra, Luis Alejandro Pérez y Mercedes Martínez

De abajo hacia arriba: la relación entre artesanos y diseñadores en México (México)

\title{
c)- Formación de Posgrado e Impacto curricular
}

La Directora Marina Matarrese es Docente de Posgrado en Diseño de la Facultad de Diseño y Comunicación en Maestría en Gestión del Diseño y Doctorado en Diseño y al igual que Luz del Carmen Vilchis Esquivel en su institución (UNAM), incorporan los contenidos de su investigación a sus asignaturas en posgrado.

\begin{abstract}
Research Project 8.3 presents reflections about the articulation between highly referenced designs in the identities of different groups and / or minorities, the field of design, the commercialization of objects of / with design and ethics. Hence, a prolific field opens up to reflect academically on the multiple factors that intersect this highly referenced issue in the processes of globalization and glocalization. Another important aspect is how inequality affects: economic, power, gender, in the recognition or not of authorship and when making claims and disputes for such recognitions.
\end{abstract}

Keywords: Design - Identity - Challenges - Inequality - Power - Latin America

Resumo: O Projeto de Pesquisa 8.3 apresenta reflexões sobre a articulação entre designs altamente referenciados nas identidades de diferentes grupos e / ou minorias, o campo do design, a comercialização de objetos de / com design e a ética. Assim, um campo prolífico se abre para refletir academicamente sobre os múltiplos fatores que cruzam este tema altamente referenciado nos processos de globalização e glocalização. Outro aspecto importante é como a desigualdade afeta: econômica, de poder, de gênero, no reconhecimento ou não da autoria e nas reivindicações e disputas por tais reconhecimentos.

Palavras chave: Design - Identidade - Desafios - Desigualdade - Poder - América Latina [Las traducciones de los resúmenes fueron realizadas a través de traductor automático] 